\title{
Dynamic transitions in a two dimensional associating lattice gas model
}

Cite as: J. Chem. Phys. 130, 184902 (2009); https://doi.org/10.1063/1.3129842

Submitted: 10 February 2009 . Accepted: 15 April 2009 . Published Online: 13 May 2009

Marcia M. Szortyka, Vera B. Henriques, Mauricio Girardi, and Marcia C. Barbosa

\section{ARTICLES YOU MAY BE INTERESTED IN}

Liquid polymorphism, order-disorder transitions and anomalous behavior: A Monte Carlo study of the Bell-Lavis model for water

The Journal of Chemical Physics 131, 164506 (2009); https://doi.org/10.1063/1.3253297

Dynamic transitions in a three dimensional associating lattice gas model

The Journal of Chemical Physics 132, 134904 (2010); https://doi.org/10.1063/1.3354112

Lattice model for water-solute mixtures

The Journal of Chemical Physics 145, 144501 (2016); https://doi.org/10.1063/1.4964396

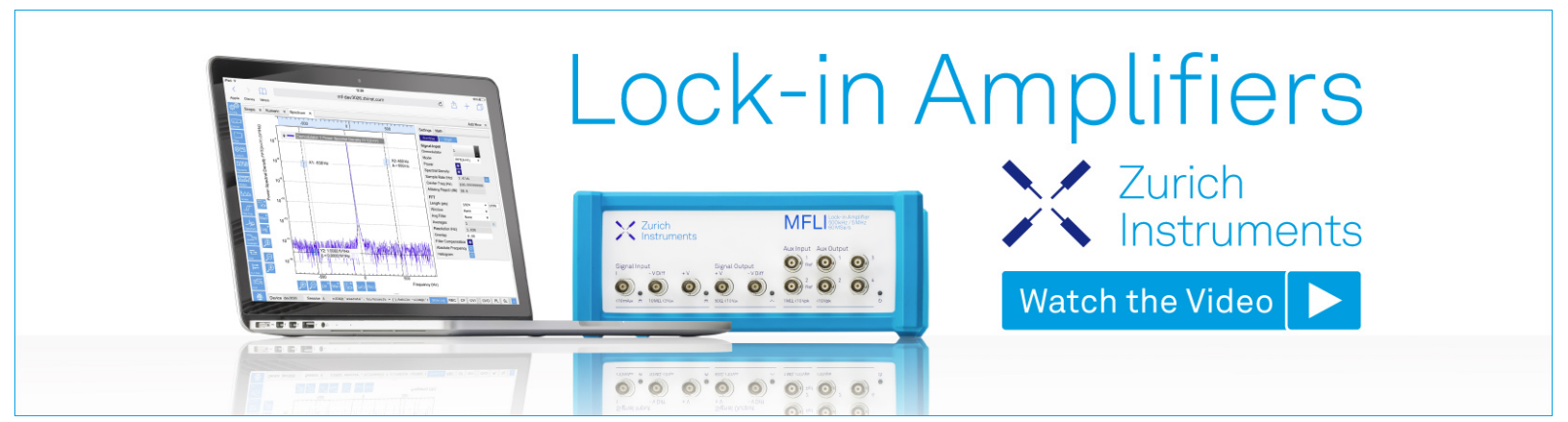

J. Chem. Phys. 130, 184902 (2009); https://doi.org/10.1063/1.3129842 


\title{
Dynamic transitions in a two dimensional associating lattice gas model
}

\author{
Marcia M. Szortyka, ${ }^{1, a)}$ Vera B. Henriques, ${ }^{2, b)}$ Mauricio Girardi, ${ }^{3, c)}$ and \\ Marcia C. Barbosa ${ }^{1, \mathrm{~d})}$ \\ ${ }^{1}$ Instituto de Física, Universidade Federal do Rio Grande do Sul, Caixa Postal 15051, Porto Alegre, \\ Rio Grande do Sul 91501-970, Brazil \\ ${ }^{2}$ Instituto de Física, Universidade de São Paulo, Caixa Postal 66318, São Paulo 05315-970, Brazil \\ ${ }^{3}$ Universidade Federal do Pampa, Caixa Postal 07, Bagé, Rio Grande do Sul 96400-970, Brazil
}

(Received 10 February 2009; accepted 15 April 2009; published online 13 May 2009)

\begin{abstract}
Using Monte Carlo simulations we investigate some new aspects of the phase diagram and the behavior of the diffusion coefficient in an associating lattice gas (ALG) model on different regions of the phase diagram. The ALG model combines a two dimensional lattice gas where particles interact through a soft core potential and orientational degrees of freedom. The competition between soft core potential and directional attractive forces results in a high density liquid phase, a low density liquid phase, and a gas phase. Besides anomalies in the behavior of the density with the temperature at constant pressure and of the diffusion coefficient with density at constant temperature are also found. The two liquid phases are separated by a coexistence line that ends in a bicritical point. The low density liquid phase is separated from the gas phase by a coexistence line that ends in tricritical point. The bicritical and tricritical points are linked by a critical $\lambda$-line. The high density liquid phase and the fluid phases are separated by a second critical $\tau$-line. We then investigate how the diffusion coefficient behaves on different regions of the chemical potential-temperature phase diagram. We find that diffusivity undergoes two types of dynamic transitions: a fragile-to-strong transition when the critical $\lambda$-line is crossed by decreasing the temperature at a constant chemical potential; and a strong-to-strong transition when the critical $\tau$-line is crossed by decreasing the temperature at a constant chemical potential. (C) 2009 American Institute of Physics.
\end{abstract}

[DOI: $10.1063 / 1.3129842$ ]

\section{INTRODUCTION}

The study of the properties of supercooled water is motivated by its well known anomalous thermodynamic behavior. Besides the density anomaly, the response functions for water appear to diverge at a singular temperature $T_{c}$ $=228 \mathrm{~K} .{ }^{1}$ This apparent divergence of the response functions led to the hypotheses of the existence of liquid polymorphism and of a second critical point, at $T_{c}=228 \mathrm{~K}^{2}$ In spite of the enormous attention given to this possible singularity, as well as to the many other anomalies, no unique explanation has yet been established. The hypothetical singular point is hidden below the homogeneous nucleation temperature $T_{H}=235 \mathrm{~K}$ (Ref. 3) in an experimentally inaccessible temperature range for bulk supercooled water. This rules out direct experimental investigation of this region in order to confirm the existence of liquid-liquid coexistence. In order to circumvent this difficulty, it has been proposed, recently, that a dynamic crossover of the transport properties such as the self-diffusion constant, $D$, and the viscosity, $\eta$, at temperatures above $T_{c}$, would indicate the presence of a critical point. ${ }^{4,5}$ The dynamic crossover has also been associated with liquid-liquid transitions in silicon ${ }^{6}$ and in nontetrahedral liquids. $^{7}$

\footnotetext{
${ }^{a)}$ Electronic mail: marcia.szortyka@ufrgs.br.

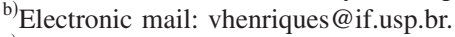

${ }^{c)}$ Electronic mail: mauricio.girardi@unipampa.edu.br.

d) Electronic mail: marcia.barbosa@ufrgs.br.
}

The basic surmise behind the link between the dynamic crossover and the presence of a second critical point goes as follows. The liquid-liquid coexistence line that separates two liquid phases terminates at a critical point. Beyond this point, at which the response functions diverge, one finds lines of maxima of these functions which asymptotically approach the critical point. This extension of the first-order phase boundary into the one-phase region has been called Widom line $^{7}$ at $T_{L}(P)$. Even though this line does not exhibit any thermodynamic transition, experiments on water show that the specific heat, shear viscosity and thermal diffusivity ${ }^{8}$ exhibit a peak when crossing the Widom line. In particular, Maruyama et al. ${ }^{9}$ conducted experiments in nanoporous (to avoid homogeneous nucleation) at ambient pressure that present a peak at the constant pressure specific heat at $T_{C p}$ $=227 \mathrm{~K}$. This temperature coincides (within the experimental error bar) with that one temperature obtained by $\mathrm{Xu}$ et al. ${ }^{10} T_{C p}=225 \mathrm{~K}$ for the location of the dynamic crossover suggesting that this crossover occurs at the Widom line, confirming the presence of the second critical point.

The presence of a peak in the specific heat in a certain region of the pressure-temperature phase diagram is not exclusivity of Widom lines. For instance, in glass formers an abrupt heat capacity drop is observed when ergodicity is broken. This change can happen very sharply in the case of fragile liquids or it may take tens of degrees in the case of strong liquids. Examples of fragile liquids are toluene and metallic systems, while covalent and network forming sys- 
tems are strong liquids. ${ }^{11}$ In the last case, the increase in the specific heat can be simply a smeared peak, located above the melting temperature, $T_{m}$, like in the case of $\mathrm{SiO}_{2}$ and of $\mathrm{BeF}_{2}{ }^{12-17}$ In the case of strong liquids, it is also possible to observe a weak transition at a temperature between the glass transition temperature, $T_{g}$, and the melting temperature, $T_{m}$. This peak in the specific heat curve occurs in the tail of a $\lambda$ thermodynamic transition where there is a little heat capacity to loose. ${ }^{18}$ Example of such strong liquids are the tetrahedral bonded liquids such as water, $\mathrm{Si}$ and Ge. This implies that observing a fragile-to-strong crossover in a region where the specific heat grows does not univocally imply the presence of a critical point. An interesting question, however, would be: does the presence of criticality result in a fragile-tostrong crossover?

In order to address this point, in this paper we analyze a model that exhibits two different critical lines and we explore what happens with the dynamics close to these line, in order to test if a fragile-to-strong transition would be a signature for criticality. The present model is an associating lattice gas (ALG) (Henriques and Barbosa) that corresponds to a lattice gas with hydrogen bonds represented through ice variables. A competition between the filling up of the lattice and the formation of an open four-bonded orientational structure is naturally introduced in terms of the ice bonding variables, and no ad hoc addition of density or bond strength variations is needed. Besides the gas phase and as a result of this competition, the model exhibits two liquid phases that bare resemblance to the two liquid phases predicted for water, corresponding to a low density liquid phase and a high density liquid phase. Moreover, it has both the diffusion and the density anomalies present in water. ${ }^{19}$

Here, the model phase diagram is reviewed and analyzed for the presence of dynamic transitions. Two new critical lines were found beyond the liquid-liquid coexistence line. We searched for fragile-to-strong transitions in the proximity of these two lines. Comparison between the behaviors of the specific heat and of the diffusion constant in these regions may help in understanding if the type of dynamic transition observed in confined water necessarily means the presence of criticality.

The remaining of this article goes as follows. In Sec. II, the lattice model is reviewed, for clarity. In Sec. III, results for the chemical potential-temperature phase diagram are shown and discussed. Our investigation of diffusion is presented in Sec. IV. Section V is a final section of conclusions.

\section{THE MODEL}

We consider a two dimensional lattice gas model of size $L^{2}$ on a triangular lattice as introduced by Henriques and Barbosa. ${ }^{20}$ In this model, particles are represented by an occupational variable, $\sigma_{i}$, which assumes the value $\sigma_{i}=0$, if the site is empty, or $\sigma_{i}=1$, if the site is full, and six orientational variables, $\tau_{i}^{A}$, that represent the different orientations that the particle might exhibit. If two neighboring sites have complementary orientations, a hydrogen bond is formed. Four bonding variables are the ice bonding arms: two donors, with $\tau_{i}^{A}$ $=1$, and two acceptors, with $\tau_{i}^{A}=-1$. The other two arms,

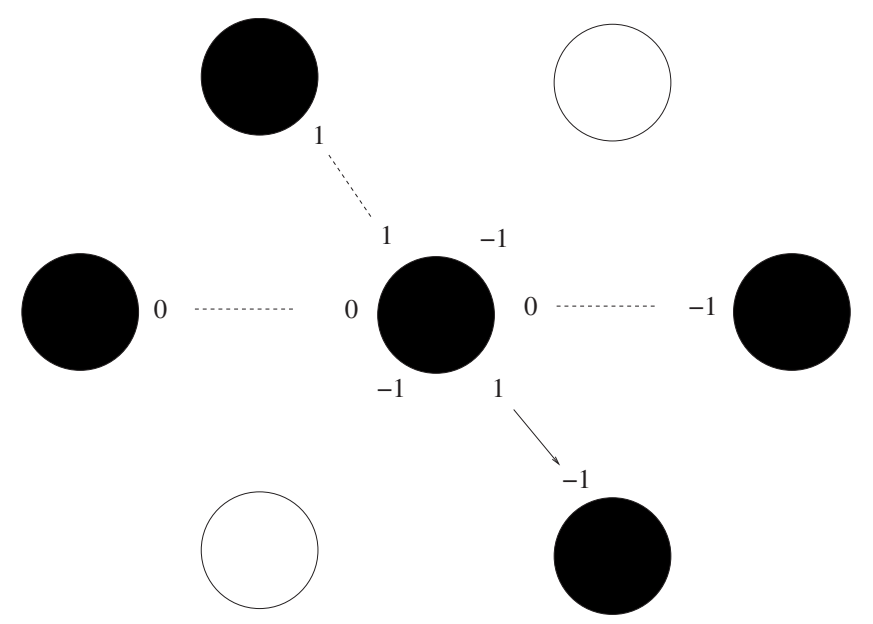

FIG. 1. Particles in the model: An occupied central site $i$ and its six bond variables, $\tau_{i}^{A}$, with $A=1, \ldots, 6$. If $\tau_{i}^{A}=0$ no bond is formed in spite of the configuration of the arm of the neighbor site. If $\tau_{i}^{A}= \pm 1$ and the neighbor's arm is $\tau_{k}^{B}=\mp 1$, a bond is formed. Dashed lines represent a nonbonding configuration, while the solid line represents a bonding configuration.

with $\tau_{i}^{A}=0$, do not form bonds, and are taken always opposite to each other, as illustrated in Fig. 1 There is no restriction for donor/acceptor arms positions, thus there are eighteen possible states for each occupied site.

The Hamiltonian includes two contributions: an isotropic, van der Waals like interaction, while the second interaction depends on the orientational degrees of freedom. Two neighboring sites, $i$ and $k$, with pointing arms $A$ and $B$, form a hydrogen bond if the product between their orientational variables is given by $\tau_{i}^{A} \tau_{k}^{B}=-1$, yielding an energy per site $e=E / L^{2}=-v$. For a nonbonding pair of occupied sites, the energy per site is $e=-v+2 u$, for $u>0$. In spite of the fact that each molecule may have six neighbors, only four hydrogen bonds per particle are allowed. The overall energy of the system is given by

$$
\mathcal{H}=(-v+2 u) \sum_{\langle i, k\rangle} \sigma_{i} \sigma_{k}+u \sum_{\langle i, k\rangle} \sigma_{i} \sigma_{k}\left[\left(1-\tau_{i}^{A} \tau_{k}^{B}\right) \tau_{i}^{A} \tau_{k}^{B}\right]
$$

where $\sigma_{i}=0,1$ are occupation variables, $\tau_{i}^{A}=0, \pm 1$ represent the arm state variables, the summation $\langle i, k\rangle$ is over neighboring sites.

Comparing the energies of the model at zero temperature two liquid phases, a low density liquid (LDL) and high density liquid (HDL) phase are found, besides the gas phase. Figures 2(a) and 2(b) illustrates the HDL and LDL phases. For high values of the chemical potential the lattice is fully occupied (density $\rho=1$ ) and the energy per site is $e=-3 v$ $+2 u$. At lower values of the chemical potential, $\mu$, the soft core repulsion becomes dominant, and the lattice becomes $3 / 4$ filled, with density $\rho=0.75$ and energy per site $e=-\frac{3}{2} v$. Like every other lattice gas, the model exhibits a gas phase, at very low chemical potentials.

At zero temperature, the grand potential per site, $\phi$ $=\Phi / L^{2}$, is given by 


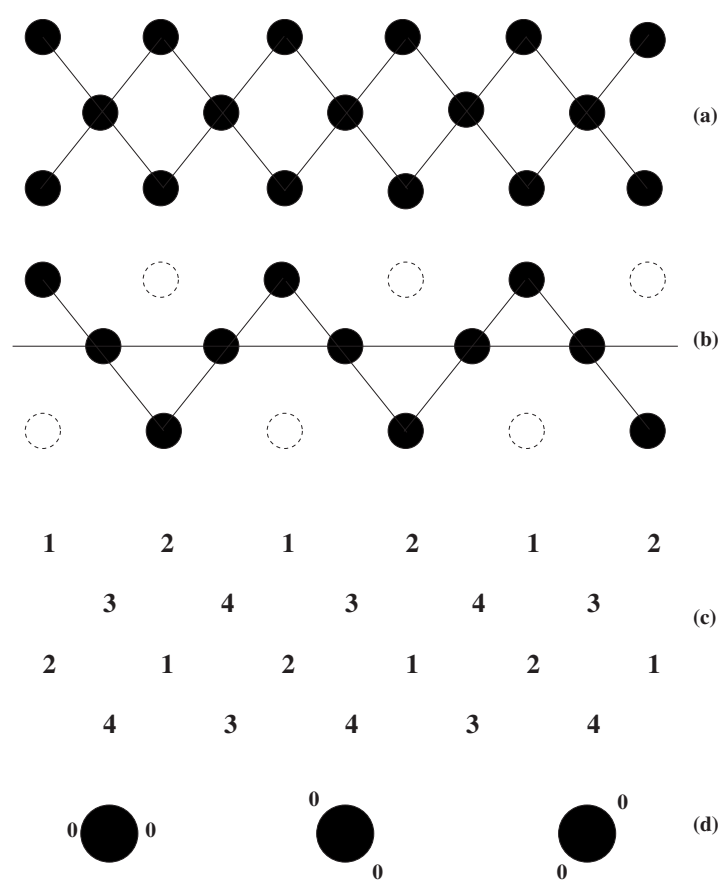

FIG. 2. (a) In the high density phase, HDL, the lattice is full, and an energy punishment arises, because two inert arms point to filled sites. Inert arms are in direction $a$. (b) In the low density phase, LDL, the lattice is $3 / 4$ filled and particles are distributed over the lattice in such a way that the inert arms point only to the empty sites. There is no energy punishment, in this case. Positional order on sublattices (1, 2, 3, and 4) is indicated. (c) Four sublattices. (d) Directions of the inert arms

$$
\phi(T=0)=\left\langle\mathcal{H}-\mu \sum_{i} \sigma_{i}\right\rangle=E-\mu N
$$

By equating the grand potential of different phases, we find that the high density phase (HDL) coexists with the low density phase (LDL) at the reduced chemical potential $\bar{\mu}$ $=\mu / v=-6+8 u / v$. The coexistence between the LDL and the gas phases occurs at $\mu / v=-2$.

The properties of the system at finite temperatures were obtained from Monte Carlo simulations in the grand canonical ensemble, through the Metropolis algorithm. We present a detailed study of the model system, for $L=30$. Some finite size scaling analysis was also undertaken, when necessary. Interaction parameters were fixed at $u / v=1$, which corresponds to "repulsive" van der Waals interaction. Reduced parameters are defined by

$$
\begin{aligned}
& \bar{T}=\frac{k_{B} T}{v}, \\
& \bar{\mu}=\frac{\mu}{v} .
\end{aligned}
$$

Equilibrium transitions were investigated through analysis of the specific heat, cumulant of energy, and the order parameters. First order transition points were located from hysteresis of the system density as a function of the chemical potential. The constant volume specific heat was calculated from simulation data obtained at constant chemical potential through the expression

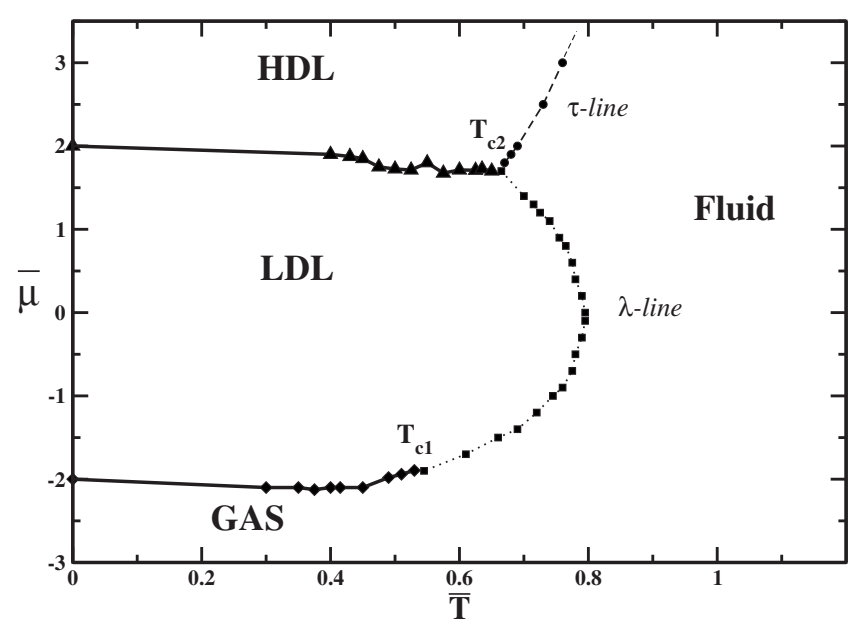

FIG. 3. Phase diagram showing reduced chemical vs reduced temperature. The diamonds represent the gas-LDL coexistence line. The triangles indicate the LDL-HDL coexistence line. $T_{c 1}$ is the tricritical point gas-LDL and $T_{c 2}$ is the tricritical point LDL-HDL. The squares and circles are lines, obtained by the maximum in specific heat, which separates fluid phase from LDL and HDL phases, respectively. The zero temperature points, at $\bar{\mu}=-2$ and $\bar{\mu}=2$, are exact.

$$
c_{V}=\frac{1}{k_{B} T^{2} V}\left(\left\langle\delta \phi^{2}\right\rangle_{\mu V T}-\frac{\langle\delta \phi \delta \rho\rangle_{\mu V T}^{2}}{\left\langle\delta \rho^{2}\right\rangle_{\mu V T}}\right)
$$

adapted from Ref. 21 to the lattice. $\rho$ is the density, $V$ is the volume and $\delta X=X-\langle X\rangle$ with $X=\phi, \rho$.

\section{THE PHASE DIAGRAM}

The chemical potential-temperature phase diagram of the model was partially analyzed in previous work, ${ }^{20}$ which focused on the coexistence lines between the low and high density liquids. In this paper the $\bar{\mu}-\bar{T}$ phase diagram is complemented by the analysis of the region beyond the coexistence line.

The complete $\bar{\mu}-\bar{T}$ phase diagram is illustrated in Fig. 3 and goes as follows. At low reduced chemical potentials, $\bar{\mu}$, for all reduced temperatures, $\bar{T}$ only the gas phase is present. As the reduced chemical potential increases a low density liquid phase appears. This phase coexists with the gas phase along a first-order transition line at $\bar{\mu}=\bar{\mu}_{\text {gas-LDL }}(\bar{T})$. For even higher reduced chemical potentials a high density liquid phase emerges. This phase coexists with the low density liquid phase at the first-order line $\bar{\mu}=\bar{\mu}_{\text {LDL-HDL }}(\bar{T})$.

However what happens at the end of the two first-order lines? In order to answer the question, we have examined the specific heat at constant volume, $c_{V}$, as a function of temperature, for fixed values of $\bar{\mu}$, in two regions of the $\bar{\mu}-\bar{T}$ phase diagram: between the two coexistence lines and above the LDL-HDL coexistence line. The results are illustrated in Figs. 4 and 5 for lattice sizes $L=10,20,30,40,50,80$, and 100.

Between the two coexistence lines, at $\bar{\mu}=0, c_{V}$ has a peak at a reduced temperature $\bar{T}=\bar{T}_{\lambda} \approx 0.79$ that diverges as $L \rightarrow \infty$. Similar behavior was observed for every investigated chemical potential between the two coexistence lines, indicating the presence of a critical line. We called this line $\lambda$ 


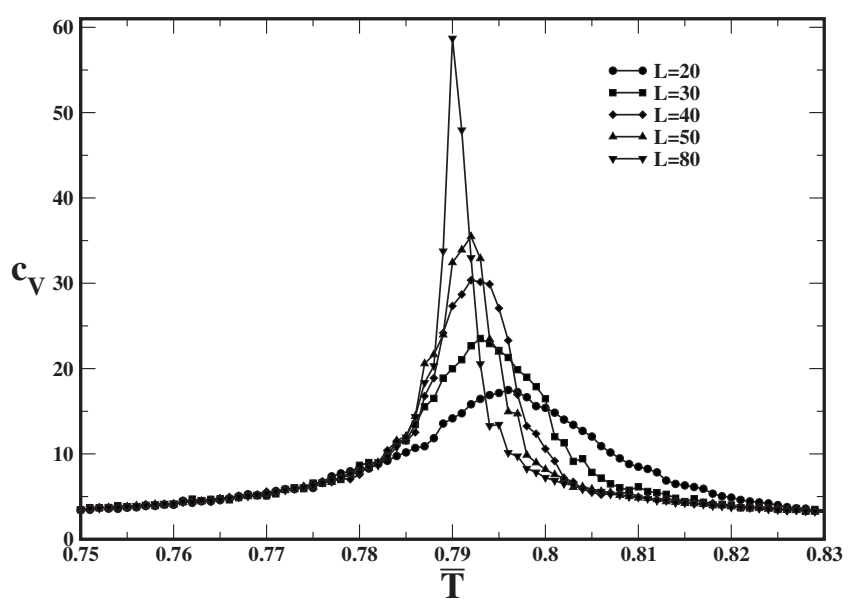

FIG. 4. Specific heat at constant volume for different lattice sizes vs reduced temperature for the $\lambda$-line $(\bar{\mu}=0)$.

and represented it in Fig. 3 through a dotted line and square symbols. Above the liquid-liquid coexistence line, for $\bar{\mu}$ $=2.5$, the specific heat, $c_{V}$, displays also a peak at $\bar{T}=\bar{T}_{\tau}$ $\approx 0.71$ that increases mildly with $L$. We have examined a range of chemical potentials above the LDL-HDL coexistence line. A line of maxima of these peaks, named $\tau$ was added to the $\bar{\mu}-\bar{T}$ phase diagram, as shown in Fig. 3 (dashed line and circles).

The criticality of $\lambda$ and $\tau$ was investigated by calculating the energy cumulant given by ${ }^{22}$

$$
V_{L}=1-\frac{\left\langle(\mathcal{H}-\langle\mathcal{H}\rangle)^{4}\right\rangle}{3\left\langle(\mathcal{H}-\langle\mathcal{H}\rangle)^{2}\right\rangle^{2}} .
$$

Figure 6 illustrates the energy cumulant for $\bar{\mu}=0$, showing a signature for criticality. Figure 7 shows the energy cumulant for $\bar{\mu}=2.5$ that also indicates the presence of criticality.

In the attempt to understand the differences between the two transitions, it is important to establish what is the structural difference between the LDL, HDL, the high and the low densities fluid phases. In order to answer this question it is necessary to investigate possible order parameters.

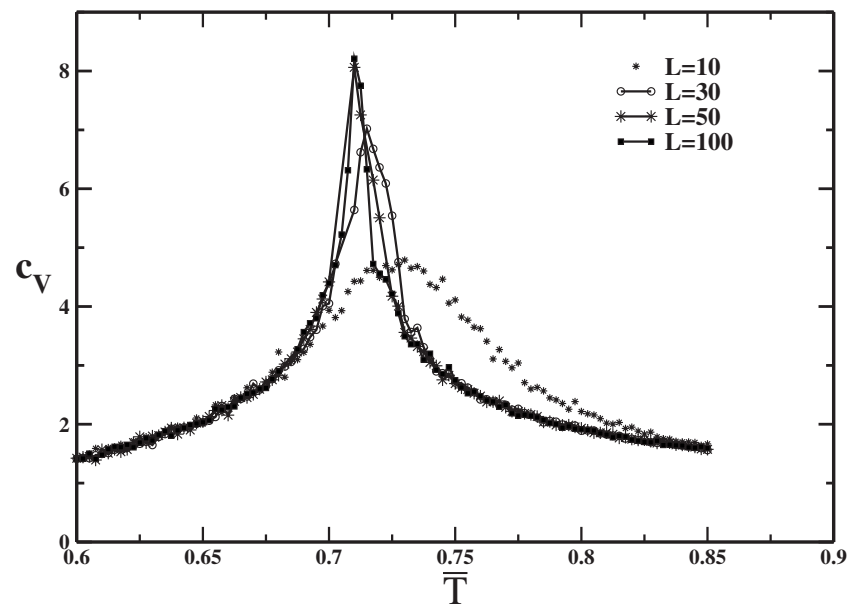

FIG. 5. Specific heat at constant volume for different lattice sizes vs reduced temperature for the $\tau$-line $(\bar{\mu}=2.5)$.

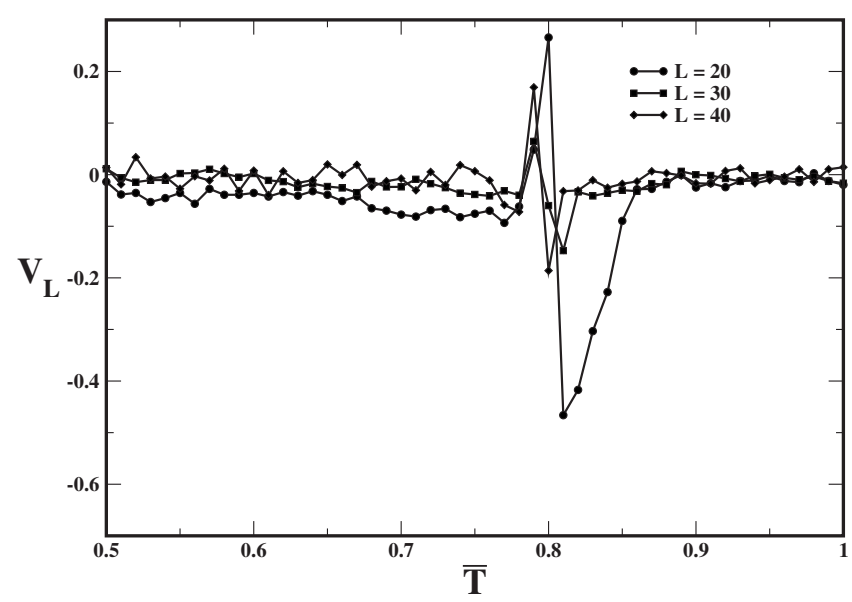

FIG. 6. Energy cumulant vs reduced temperature for the $\lambda$-line at $\bar{\mu}=0$.

We first look at particle density, $\rho$, and the number of hydrogen bonds per particle, $\rho_{h b}$. Figures 8 and 9 shows an important difference in behavior of these properties on crossing the two transitions, the $\lambda$ and the $\tau$ transition. In the case of the $\lambda$ line (circles), as temperature is decreased toward the specific heat peak position, density increases abruptly toward $\rho=0.75$. As the transition is approached, the system orders itself by forming hydrogen bonds and by releasing nonbonded particles. A density maximum must occur on the other side of the transition, at a higher temperature. On the other hand, in the case of the $\tau$ line (squares), both density and number of bonding particles increase smoothly as the temperature decrease toward the specific heat peak temperature. Thus, in this case, bonding and lattice filling occur simultaneously. No density maximum is expected in this region of the phase diagram.

In order to better characterize the structure of the liquid in each phase, we also look at sublattice properties. The LDL is characterized at low temperatures by the structure illustrated in Fig. 2. This suggests that the $\lambda$ transition might be better understood in terms of the density of the sublattices shown in Figs. 2(c). Since at zero temperature one of the sublattices is empty (sublattice 2 in the figure), the order parameter should be related to the density of the empty sublattice, namely,

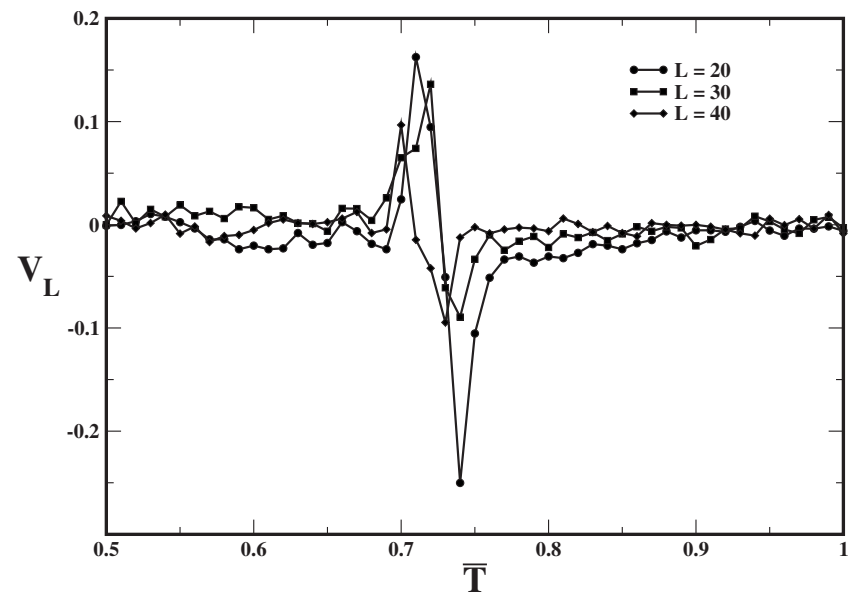

FIG. 7. Energy cumulant vs reduced temperature for the $\tau$-line at $\bar{\mu}=2.5$. 


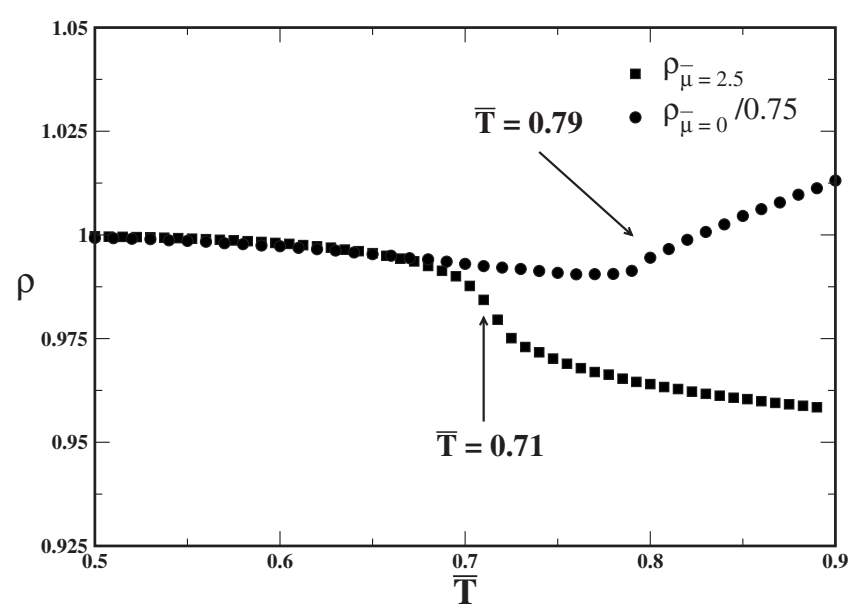

FIG. 8. Reduced density $\rho / 0.75$ for the $\lambda$-line $(\bar{\mu}=0)$ (full circles) and density $\rho$ for the $\tau$-line $(\bar{\mu}=2.5)$ as functions of the reduced temperature.

$$
\theta_{\lambda}=1-\frac{\rho_{\text {empty }}}{\rho},
$$

where $\rho_{\text {empty }}$ is the density of the emptiest sublattice and $\rho$ is the overall density. The result is illustrated in Fig. 10 for reduced chemical potential $\bar{\mu}=0$, at which the $\lambda$-line is crossed. The transition is characterized by three sublattices becoming full, while the fourth one becomes empty. Thus the LDL phase may be described as an ordered phase both with respect to position and to orientation of the particles.

In the case of the HDL phase, all the sublattices are full at zero temperature, as shown in Fig. 2. Thus, for the $\tau$ transition, $\theta_{\lambda}$, given by Eq. (7), is not a good order parameter. On the other side, in the ground state of the HDL phase, all particles display inert arms in the same direction, as shown in Fig. 2. Therefore, the order parameter for the $\tau$ transition should be related to the number of inert arms in the direction they order at zero temperature, $n_{0}$, namely,

$$
\theta_{\tau}=\frac{3}{2}\left(\frac{n_{0}}{\rho}-\frac{1}{3}\right)
$$

where the direction of inert arms is defined in Fig. 2(d) and $\rho$ is the density of the system. The subtraction of $1 / 3$ guaran-

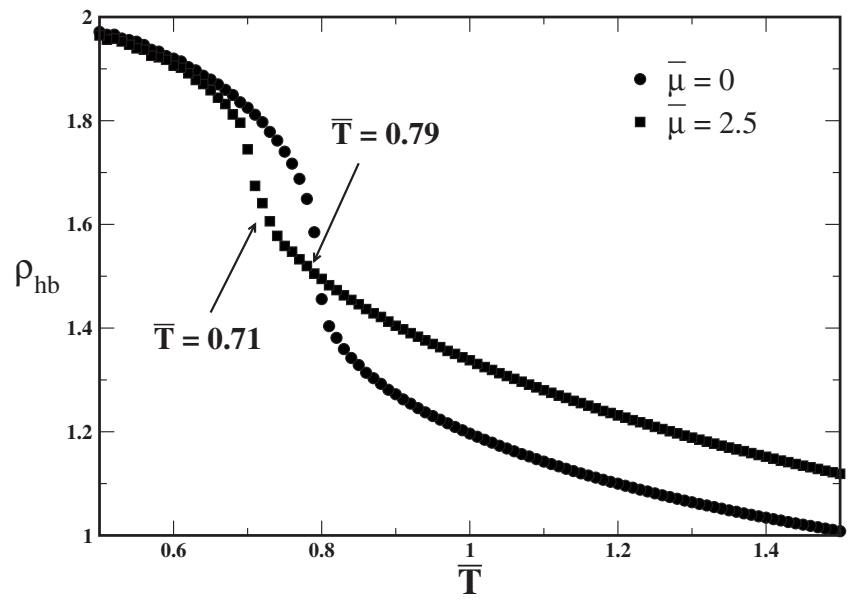

FIG. 9. Number of hydrogen bonds per particle as a function of the reduced temperature for the $\lambda$ and $\tau$ lines. Symbols and numbers as in previous figures.

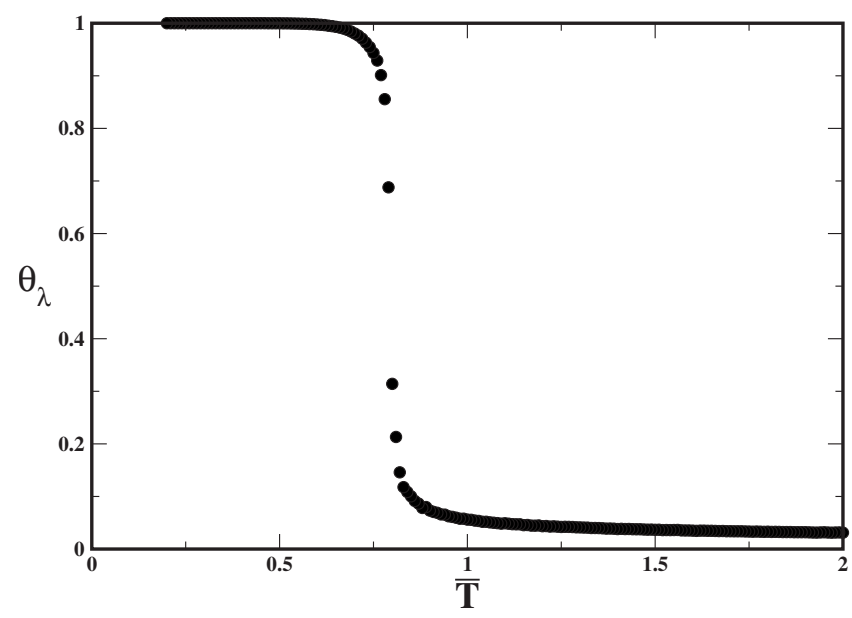

FIG. 10. Order parameter, $\theta_{\lambda}$, vs reduced temperature for reduced chemical potential $\bar{\mu}=0$.

tees that at high temperatures where the three arm states should be equivalent, $\theta_{\tau}$ is zero. The order parameter behavior, $\theta_{\tau}$ versus temperature, illustrated in Fig. 11 shows that the system actually tends to have particles with inert arms in a specific direction, as the temperature is decreased. This allows us to interpret the HDL phase as an ordered phase with respect to orientation of the particles.

Analysis of the order parameters indicates that both transitions may be described as order-disorder transitions. However, the $\lambda$-transition corresponds to ordering of position (accompanied by bond ordering), whereas the $\tau$-transition corresponds only to bond ordering.

\section{DYNAMICS}

In order to quantify mobility in supercooled liquids, the concept of fragility was introduced by Angell. ${ }^{23}$ Analyzing relaxation as a function of temperature, liquids are classified as strong, when relaxation follows an Arrhenius law, or fragile, when the relaxation follows a non-Arrhenius law. Strong liquids present structure that is preserved when temperature is increased, whereas in fragile liquids this structure is easily broken, as temperature increases.

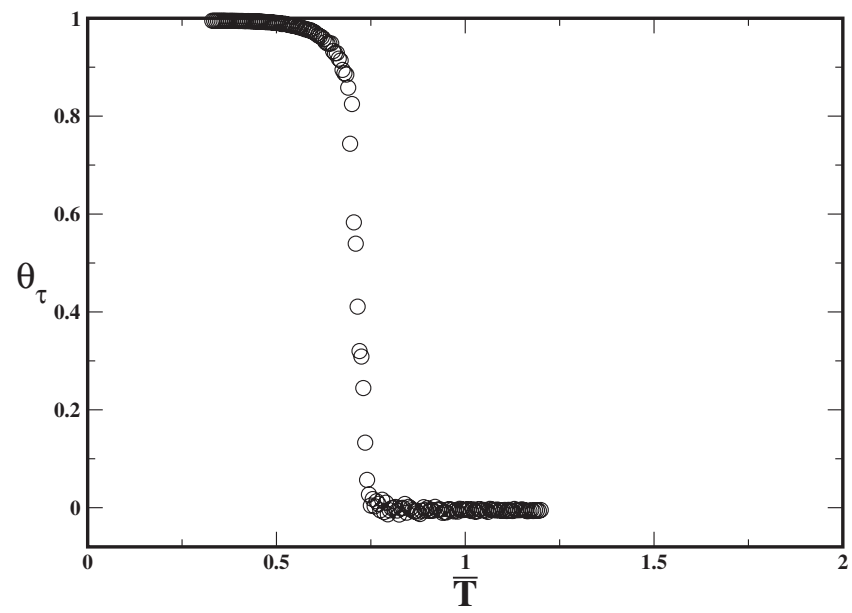

FIG. 11. Order parameter, $\theta_{\tau}$, vs reduced temperature across the $\tau$-line $(\bar{\mu}$ $=2.5$ ). 
Within the framework of the Adam-Gibbs theory, ${ }^{24}$ viscous liquids are described as being made of clusters that rearrange cooperatively in order to pass through the free energy barrier. Consequently, diffusion depends on this cooperative rearrangement of the clusters through equation

$$
D=D_{0} \exp \left(\frac{C \Delta \mu}{T S_{c}}\right),
$$

for the diffusion constant $D$. Here $D_{0}$ and $C$ are constants, $\Delta \mu$ is the free energy barrier which the clusters have to overcome. $S_{c}$ is the configurational entropy, given by

$$
S_{c}(T)=\int_{T_{K}}^{T}\left(\frac{\Delta c_{p}}{T}\right) d T,
$$

that describes how the structure of the liquid changes with temperature. In Eq. (10) $T_{K}$ is the Kauzmann temperature ${ }^{23}$ [for which $S_{c}\left(T_{K}\right)=0$ ] and $\Delta c_{p}$ is the difference in specific heat between the crystal and the liquid configurations, at temperature $T$. If $S_{c}$ is temperature independent, the diffusion follows an Arrhenius law, the liquid is very structured and the system is a strong liquid. If the configurational entropy depends on temperature, $S_{c}=\Delta C_{p} \ln T / T_{k}$, Eq. (9) becomes a Vogel-Fulcher equation, the liquid is not structured and is classified as a fragile liquid.

Now we investigate the dynamic properties on crossing the $\lambda$ and the $\tau$ lines, at constant chemical potential (see Fig. 3 ), by analyzing behavior of model diffusivity. In order to compute diffusion coefficient we first equilibrate the system at fixed chemical potential and temperature. In equilibrium this system has $n$ particles. Starting from this equilibrium configuration at a time $t=0$, each one of these $n$ particles is allowed to move to an empty neighbor site randomly chosen. The movement is accepted if the total energy of the system is reduced, otherwise it is accepted with a probability $\exp \left(\Delta E / k_{B} T\right)$ where $\Delta E$ is the difference between the energy of the system after and before the movement. After repeating this procedure $n t$ times, the mean square displacement per particle at a time $t$ is given by

$$
\left\langle\Delta r(t)^{2}\right\rangle=\left\langle(\mathbf{r}(t)-\mathbf{r}(0))^{2}\right\rangle,
$$

where $\mathbf{r}(0)$ is the particle position at the initial time and $\mathbf{r}(t)$ is the particle position at a time $t$. In Eq. (11), the average is taken over all particles and over different initial configurations. The diffusion coefficient is then obtained from Einstein's relation

$$
D=\lim _{t \rightarrow \infty} \frac{\left\langle\Delta r(t)^{2}\right\rangle}{4 t} .
$$

Since the time is measured in Monte Carlo time steps and the distance in number of lattice distance, a dimensionless diffusion coefficient is defined as

$$
\bar{D}=\lim _{t \rightarrow \infty} \frac{\left\langle\Delta \bar{r}(t)^{2}\right\rangle}{4 \bar{t}},
$$

where $\bar{r}=r / a$ and $a$ is the distance between two neighbor sites and $\bar{t}=t / t_{\mathrm{MC}}$ is the time in Monte Carlo steps.

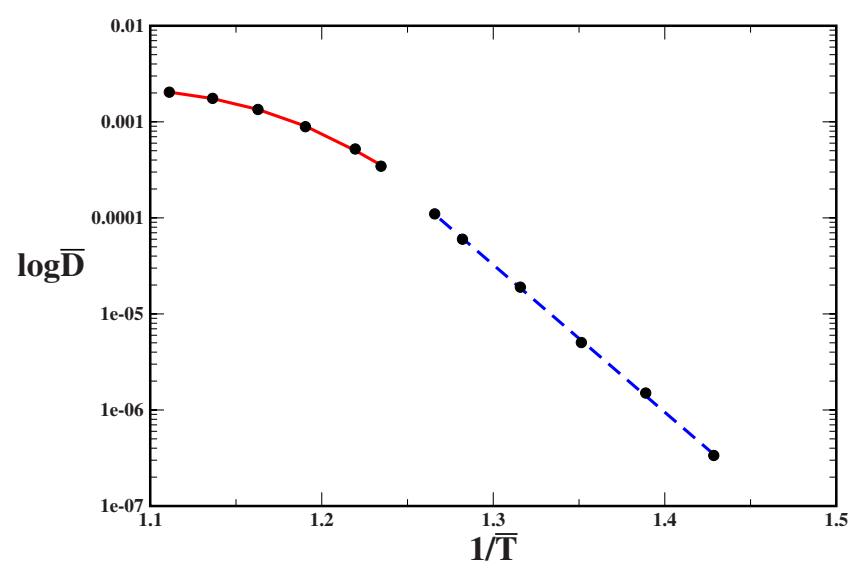

FIG. 12. (Color online) Logarithm of diffusion constant vs inverse temperature across the $\lambda$-line $(\bar{\mu}=0)$. Symbols are diffusion coefficient measured in simulation, solid line is a cubic fit and dashed line is an exponential fit. At high temperatures system behaves as a fragile liquid following a nonArrhenius law, while for low temperatures the system behaves like a strong liquid following an Arrhenius law.

Figure 12 illustrate the behavior of the diffusion coefficient $D$ with the inverse of the reduced temperature $1 / \bar{T}$, for reduced chemical potential $\bar{\mu}=0$. At higher temperatures, diffusivity follows a non-Arrhenius trend, namely,

$$
y=A_{0}+A_{1} x+A_{2} x^{2}+A_{3} x^{3}
$$

indicating that the low density disordered fluid phase is a fragile liquid. At lower temperatures, diffusivity displays Arrhenius behavior, given by

$$
y=A_{0} \exp \left(-\frac{A_{1}}{x}\right)
$$

thus characterizing the low density ordered liquid phase as a strong liquid. $A_{i}$ are fitting parameters in both equations.

This change in dynamics over the critical $\lambda$ line occurs because the liquid is structurally different on both sides of the critical line. In the low density disordered fluid phase, interstitial particles weaken the hydrogen bonds and disrupt the network, so particles can rearrange fast and the process of diffusion is not energy activated. In the LDL phase, the network is fully developed, resulting in an ordered liquid, in which particles are "trapped," increasing relaxation time and characterizing this phase as a strong liquid, in which an energy activated diffusion process takes place. This is the dynamic transition observed when crossing a Widom line in ramplike models, ${ }^{10,25}$ which suggests that the dynamic transition is not linked with the type of line but with the structuring of the system if this happens with or without a thermodynamic phase transition.

Since the HDL is also a structured phase, in principle, a fragile-strong transition in the dynamics of diffusion could also be expected on crossing the $\tau$ line. However, this is not the case. Figure 13 illustrate the behavior of the diffusion constant as function of inverse temperature, $1 / \bar{T}$, for fixed reduced chemical potential $\bar{\mu}=1.85$. At higher temperatures and high chemical potentials (or equivalently high densities), the fluid phase has an Arrhenius behavior and so it is a strong liquid. At lower temperatures, the HDL phase also displays 


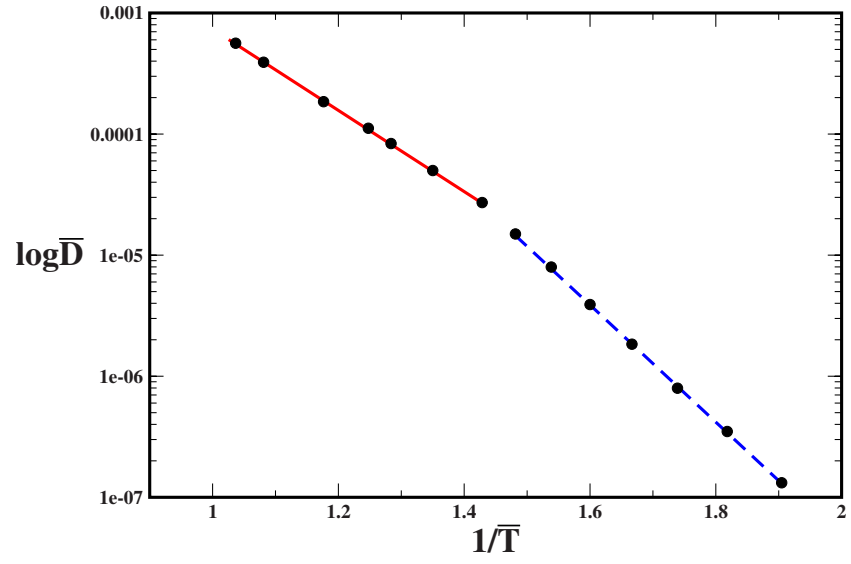

FIG. 13. (Color online) Logarithm of diffusion constant vs inverse temperature across the $\tau$-line $(\bar{\mu}=2.5)$. Symbols are diffusion coefficient measured in simulation and solid and dashed lines are two different exponential fits. In the region of $\tau$ line system behaves as a strong liquid in both sides. The difference between the two Arrhenius behaviors is the activation energy, defines the slope of the curve.

an Arrhenius behavior, and therefore is also a strong liquid. The HDL phase and high density fluid phases are both strong liquids that differ in the activation energy. In resume, when the system crosses the $\tau$ line, we have a dynamic transition, and a strong-strong crossover is observed. In this case, the activation energy of the HDL phase is higher than the activation energy of the high density fluid phase, indicating that the HDL phase is more ordered than the high density fluid phase. Diffusion is lowered in the HDL phase because particles spend more time trying to rearrange, in comparison with the high density fluid phase.

How can we explain the existence of a fragile-to-strong crossover on the critical $\lambda$-line and a strong-to-strong transition on the $\tau$ line? The answer is given by the structure of the liquid, described in the previous section. On crossing the $\lambda$-line, the hydrogen-bonded net breaks down abruptly, while the $\tau$-line is accompanied by a much smoother melting of the H-bond network.

\section{CONCLUSIONS}

In this paper we have analyzed equilibrium and dynamic properties of the ALG model, a lattice gas with hydrogen bonds represented through ice variables. Competition between the filling up of the lattice and the formation of an open four-bonded orientational structure leads to the presence of two liquid phases and a gas phase. The coexistence lines between the LDL and the gas phases, and between the LDL and HDL phases are connected by a critical $\lambda$-line. Besides the $\lambda$-line, a second one, the $\tau$-line, also emerges from the LDL-HDL coexistence line. This line is also identified by a peak in the specific heat.

The system undergoes two kinds of dynamic transitions: a fragile-to-strong transition, on crossing the $\lambda$-line, and a strong-to-strong transition, on crossing the $\tau$-line. Both dynamic transitions are related with changes in the position and orientational structure of the system. In the fragile-to-strong case, upon crossing the $\lambda$-line toward higher temperatures, the system undergoes a positional order-disorder transition, accompanied by an orientational transition. On crossing the $\tau$-line, the strong-to-strong dynamic transition is companion to an order-disorder equilibrium transition with respect to particle orientation, which bares no relation to a density anomaly.

Our results point out in the direction that criticality does not necessarily means fragile-strong transition. This change is, in fact, related to the change in structure that in the present case appears in two very different forms.

\section{ACKNOWLEDGMENTS}

This work was supported by the Brazilian science agencies CNPq, FINEP, Capes, and Fapesp.

${ }^{1}$ R. J. Speedy and C. A. Angell, J. Chem. Phys. 65, 851 (1976).

${ }^{2}$ P. H. Poole, F. Sciortino, U. Essmann, and H. E. Stanley, Nature (London) 360, 324 (1992).

${ }^{3}$ D. H. Rasmussen and A. P. MacKenzie, Interactions in the WaterPolyvinylpyrrolidone System at Low Temperatures (Plenum, New York, 1972).

${ }^{4}$ L. Liu, S.-H. Chen, A. Faraone, S.-W. Yen, and C.-Y. Mou, Phys. Rev. Lett. 95, 117802 (2005).

${ }^{5}$ S.-H. Chen, F. Mallamace, C.-Y. Mou, M. Broccio, C. Corsaro, A. Faraone, and A. L. Liu, Proc. Natl. Acad. Sci. U.S.A. 103, 12974 (2006).

${ }^{6}$ S. Sastry and C. A. Angell, Nature Mater. 2, 739 (2003).

${ }^{7}$ L. M. Xu, I. Ehrenberg, S. V. Buldyrev, and H. E. Stanley, J. Phys.: Condens. Matter 18, S2239 (2006).

${ }^{8}$ M. A. Anisimov, J. V. Sengers, and J. M. H. Levelt Sengers, Aqueous Systems at Elevated Temperatures and Pressures: Physical Chemistry in Water Steam and Hydrothermal Solutions (Elsevier, The Netherlands, 2004).

${ }^{9}$ S. Maruyama, K. Wakabayashi, and M. A. Oguni, AIP Conf. Proc. 708, 675 (2004).

${ }^{10}$ L. Xu, P. Kumar, S. V. Buldyrev, S.-H. Chen, P. Poole, F. Sciortino, and H. E. Stanley, Proc. Natl. Acad. Sci. U.S.A. 102, 16558 (2005).

${ }^{11}$ C. A. Angell, Science 267, 1924 (1995).

${ }^{12}$ P. Richet, Y. Bottinga, L. Denielou, J. P. Petitet, and C. Tequi, Geochim. Cosmochim. Acta 46, 2639 (1982).

${ }^{13}$ P. Richet and Y. Bottinga, C. R. Acad. Sci., Ser. II 295, 1121 (1982).

${ }^{14}$ S. Tamura, T. Yokokawa, and K. J. Niwa, J. Chem. Thermodyn. 7, 633 (1975).

${ }^{15}$ S. Tamura and T. Yokokawa, Bull. Chem. Soc. Jpn. 48, 2542 (1975).

${ }^{16}$ P. Scheidler, W. Kob, A. Latz, J. Horach, and K. Binder, Phys. Rev. B 63, 104204 (2001).

${ }^{17}$ R. B. Sosman, The Properties of Silica, American Chemical Society Monograph No. 27 (Chemical Catalog, New York, 1927).

${ }^{18}$ C. A. Angell, Science 319, 183 (2008).

${ }^{19}$ M. M. Szortyka and M. C. Barbosa, Physica A 380, 27 (2007).

${ }^{20}$ V. B. Henriques and M. C. Barbosa, Phys. Rev. E 71, 031504 (2005).

${ }^{21}$ M. P. Allen and D. J. Tildesley, Computer Simulations of Liquids (Clarendon, Oxford, 1987).

${ }^{22}$ S.-H. Tsai and S. R. Salinas, Braz. J. Phys. 28, 58 (1998).

${ }^{23}$ C. A. Angell, J. Res. Natl. Inst. Stand. Technol. 102, 171 (1997).

${ }^{24}$ G. Adam and J. H. Gibbs, J. Chem. Phys. 43, 139 (1965).

${ }^{25}$ L. Xu, S. Buldyrev, C. A. Angell, and H. E. Stanley, Phys. Rev. E 74, 031108 (2006). 